\title{
Lettuce prey
}

\author{
Two recent Escherichia coli outbreaks, a United States government shutdown and the imminent departure of the \\ United Kingdom from the European Union bring into focus the fragility of global food supply systems.
}

ast year, the United States saw two outbreaks of Escherichia coli O157

in romaine lettuce, the largest such events since 2006. The first outbreak in the spring saw 210 people falling ill in 36 states, resulting in five deaths and a massive recall on romaine while the Food and Drug Administration (FDA) and the Centers for Disease Control (CDC) investigated. "For three months out of the year the majority of the romaine sold in the US comes from the desert growing region of the CaliforniaArizona border" says Patrick Baur, a postdoc researcher on food safety at the University of California, Berkeley, USA. Despite this head start, investigators struggled to find either the source or cause of the outbreak. It was only after testing water samples from a 3.5-mile stretch of a nearby irrigation canal, downstream from cattle feedlots, that it was determined that the E. coli contamination came from multiple farms and went through multiple processing facilities. The FDA's report on the event came out on 1 November 2018 , by which time a second outbreak was underway, affecting 62 people in 16 states before it ended in January 2019.

It is to be celebrated that the FDA and CDC identified the location and vector of the contamination, but the incident displays a lack of risk awareness that Baur believes is endemic to the apparatus of food safety in the US. Even tracing the source of the outbreak all the way back to these farms is unlikely to solve the problem that was uncovered, as no single entity can take control and change it: "not the local irrigation system, not the livestock growers upstream, not any individual farmer", warns Baur. "We have a whole system that magnifies risk".

The US food safety system suffers from the lack of a central authority to oversee the food industry (responsibilities being split between multiple federal agencies and the 50 states), but it is also worth looking at the consolidation of the industry, as bottlenecks in the supply chains push crops from numerous farms for numerous brands through the same processing and distribution, thus making crosscontamination more likely. According to Laura DeLind and Philip Howard (Agr. Hum. Values 25, 301-317; 2008), during the 2006 E. coli outbreak, one day's worth of spinach from a single farm, for a single brand of bagged vegetables, entered a processing facility where it 'comingled' and sparked a recall of 34 brands of spinach and spinach-containing products across the US.

Such incidents bring to mind the German sociologist, Ulrich Beck, who wrote some of the most pioneering analysis of environmental risks by focusing on the nature of complexity in modern socio-technological systems. Beck's prime examples were nuclear weapons, but the theoretical framework he created can be applied to every instance of systemic risks and hidden dangers.

Complexity and large-scale risk in food safety is hardly news, but Beck showed how risks manifest and why they are so difficult to address. In Risk Society (1992), he asserted that technological and environmental dangers become democratized at larger scales, affecting wider cross sections of society due to the inability to escape the breakdowns of a system that lacks redundancy; the 2018 E. coli outbreak affected conventional and organic spinach equally. Essays in Ecological Enlightenment (1995) question the mindset of bureaucrats and engineers who, having built complex systems, assert that only their expertise and tools, which caused the risks, can solve them.

"The produce industry, food scientists and agronomists want a technological solution to solve this, like with pasteurization", says Baur. "The idea of a silver bullet for leafy greens is very compelling, ... but you'd have to selectively breed or genetically engineer some 200 crops in California alone, and you'd have to do it for multiple pathogens. Until we get better at acknowledging the risks of the system, we're going to get bigger problems."

During the second E. coli outbreak last year, Sarah Taber at Slate magazine (http://bit.ly/2Eaw0vq) suggested that the crackdown on undocumented immigrants in the US may be playing a role in recent outbreaks. Many farm and food processing workers are undocumented, but also have some of the most experience. Losing them means losing institutional memory in an already fragile and interconnected food safety system. The recent federal government shutdown did not help matters, as the FDA was forced to reallocate and prioritize investigations over inspections. The US may have dodged a bullet in regards to outbreaks during the shutdown itself, but the long-term impacts will still be felt. The system relies on daily cooperation between regulators and industry, keeping everybody abreast of environmental assessments and outbreak scenarios. This flow of information was badly damaged during the shutdown.

This is not a problem unique to the US. The United Kingdom is facing total uncertainty regarding its food importation guidelines following its anticipated departure from the European Union. Some $80 \%$ of the food safety regulations that the UK follows come from EU law, and should the UK and EU agree to a withdrawal deal, these will remain in force both for trade with the EU and imports from other countries. However, in the event of a so-called 'no-deal Brexit', in which the UK and EU fail to come to an agreement, the UK could be forced to trade with its biggest food partner on World Trade Organization terms. Such abrupt changes could disrupt the entire food system in the UK. As an island that imports well over half of its fruits and vegetables, the British government may be left scrambling to maintain this supply anyway it can, 'standards be damned'.

In their study of the 2006 spinach outbreak, Delind and Howard argued for a food system inspired by 'subsidiarity' with decisions made at the smallest possible level. They argued that government and industrial proposals for more expansive regulations put too much onus on small farmers and operations, even though these risks do not arise with them but with national and global apparatuses of collection and distribution. The record since then only proves that our fragile, highly centralized food systems are jeopardized by political and economic forces that fail to grasp the risks they create. Perhaps it is not the pathogens then that are to blame for potentially deadly outbreaks, but us.

Published online: 8 March 2019 https://doi.org/10.1038/s41477-019-0393-0 